\title{
Hypothyroidism in pregnancy - consensus on testing and treatment
}

\begin{abstract}
Maternal hypothyroidsm is relatively common but frequently undiagnosed in pregnancy. The consequences on mother and fetus are detrimental. The guidelines by ATA present a lucid explanation for diagnosis and treatment of this ailment but it has to be seen and interpreted in context to local regional demographics of population. The present paper is based on studies in literature and ATA guidelines for treatment of hypothyroidsm in preganancy.
\end{abstract}

Keywords: Maternal hypothyroidsm, Thyroid, Pregnancy
Volume 7 Issue 3 - 2019

\author{
Brinderjeet Kaur,' Rateesh Sareen ${ }^{2}$ \\ 'Assistant Consultant, Department of Obstetrics and \\ Gynecology, Santokba Durlabhji Memorial Hospital and \\ Research Center, India \\ ${ }^{2}$ Assistant Consultant, Department of Pathology, Santokba \\ Durlabhji Memorial Hospital and Research Center, \\ India
}

Correspondence: Brinderjeet Kaur, Assistant Consultant, Department of Obstetrics and Gynecology, Santokba Durlabhji Memorial Hospital and Research Center, India, Email dr.bjkaur@gmail.com

Received: May 22, 2019| Published: June 19, 2019

\section{Introduction}

Pregnancy affects thyroid physiology resulting in: increase in size (10-30\%), increase production of thyroid hormones like thyroxine (T4) \& Triiodothyronine (T3) by $50 \%$ and increase in daily Iodine requirement of Iodine by roughly $50 \%$, to the non pregnant state in woman. The physiological changes in thyroid are sine quo non of pregnancy but the pathological process may result in thyroid disease. ${ }^{1}$ One of the problematic areas in diagnostics is interpretation of thyroid function tests in pregnant woman as it differs from non pregnant state. The accurate upper reference interval of serum TSH in pregnant woman is not clearly defined because of the stimulation of thyroid hormone secretion by placental human chorionic gonadotropin (hCG) resulting in decreased maternal thyrotropin concentration and thyroid peroxidase (TPOAb) or thyroglobulin antibody $(\mathrm{TgAb})$ positivity that adversely impacts maternal thyroid status. ${ }^{2}$ Physiological changes in pregnancy cause increased renal iodine excretion, increased thyroid hormone production, increase in thyroid binding proteins and stimulation of thyroid by placental beta HCG. ${ }^{3,4}$ Thyroxine binding globulin (TBG) and total T4 (TT4) are increased in pregnancy by 7 weeks of gestation, attain peak concentration by 16 weeks and there after remain high till delivery causing reduction in serum TSH concentration. ${ }^{5}$

\section{Reference range (Strong recommendation with moderate quality evidence)}

It is recommended to use population based trimester specific reference ranges as TSH reference range vary significantly in different races, ethnicity and various geographic regions. Most of the laboratories estimate FT4 measurement by immunoassay technology using automated platforms. The unbound T4 fraction represents only $0.03 \%$ of serum TT4 content. ${ }^{4,6,7}$ FT4 is the form that is available for tissue uptake and is measured in picomolar concentrations in contrast to TT4 which is measured in nano molar range. FT4 measurement is challenging as it gets affected by binding protein levels which show physiological variations in pregnancy. Pregnancy is characterized by high concentration of TBG with Non esterified fatty acids and low albumin concentration therefore the FT4 test reference range vary widely. ${ }^{6,7}$ The various kit manufacturers emphasize on establishment of reference intervals by laboratories themselves but it is often impractical for laboratories to recruit subjects and perform the herculean task. The laboratories on the other hand adopt pregnancy related reference ranges as provided by the manufacturers of test kits which unfortunately lack adjustments as per race, ethnicity and population specific iodine uptake, all these add to uncertainty of FT4 estimate making reliance on FT4 test during pregnancy questionable. The TT4 and calculated FT4 show inverse relation with TSH in nutshell TT4 measurement is superior to immunoassay measurement of FT4. FT4 should be measured by liquid chromatography (LC) or mass spectroscopy (MS) as they are at par with measurement by classical equilibrium dialysis method. Unfortunately LC and or MS are not used by laboratories due to high instrument and operational costs. Calculated FT4 index can reliably be done using TT4 concentration and clinically acceptable upper reference range is determined by shifting the non pregnant limit 50\% higher. However, this is applicable after 16 weeks of pregnancy. Estimation of FT4 before 16 weeks can be made by increasing the upper limit of non pregnant reference limit by $5 \%$ per week of pregnancy beginning with $7^{\text {th }}$ week. ${ }^{8,9}$

\section{Defining maternal hypothyroidism}

Increase in Serum TSH level with decreased Serum FT4 concentration during pregnancy is hallmark of primary maternal hypothyroidism. The literature studies also mention that this biochemical profile is seen in healthy non pregnant women. ${ }^{10,11}$ One of the factor responsible for high TSH in non pregnant women is iodine deficiency. In that case where iodine levels are within normal range the causative etiology of hypothyroidsm is autoimmune thyroid disease. This further complicates the situation in pregnancy as high TSH levels in pregnancy are associated with detection of thyroid antibodies in 30-60\% of cases. ${ }^{11-13}$ In the 2011 American Thyroid association (ATA) guidelines, the upper reference limit for serum TSH concentration during pregnancy was defined as $2.5 \mathrm{mU} / \mathrm{L}$ in the first trimester and $3.0 \mathrm{mU} / \mathrm{L}$ in the second and third trimester respectively. These cutoffs were based on studies comprising a total cohort of about 5500 subjects. ${ }^{14-19}$ The consensus on reference intervals continues as evidenced by other studies. ${ }^{20}$ Mother and fetal complications are 
higher in TPOAb positive pregnant woman for unknown causes. ${ }^{12}$ Therefore, it is difficult to set a cut off of maternal TSH level for therapy related decision making.

\section{Pregnancy specific TSH reference range}

a. Who should define- Laboratory itself

b. Population selection- Healthy pregnant woman with adequate iodine uptake

The upper limit of TSH is $4.0 \mathrm{Mu} / \mathrm{L}$ which for most assays is nearly equal to reduction of non pregnant reference range by $0.5 \mathrm{Mu} / \mathrm{L}$. It is also suggested to screen all pregnant women for TPOAb status when $\mathrm{TSH}$ is above $2.5 \mathrm{mU} / \mathrm{L}$

\section{Adverse outcome}

Maternal hypothyroidism results in adverse pregnancy related complications ranging from premature \& low birth weight,loss of pregnancy, poor fetal neurocognitive development, lower IQ of new born. ${ }^{21,22}$ Different studies have found maternal and child complications due to maternal hypothyroidism like Abalovich et al. ${ }^{23}$ - fetal loss in $60 \%$ of subjects and Leung et al. ${ }^{24}$ - gestational hypertension in $22 \%$ patients. The association of pregnancy $\operatorname{loss}^{25}$ with maternal hypothyroidism was seen in studies by Negro et al. ${ }^{26}$ Benhadi et al. ${ }^{27}$ and Liu et al. ${ }^{28}$ Pregnancy loss is difficult to determine as many pregnancies occur before they are recognized. In study by Negro et al, higher incidence of pregnancy loss was noted in woman with TSH level $>2.5 \mathrm{Mu} / \mathrm{L}$ is compared to those with TSH levels $<2.5 \mathrm{mU} / \mathrm{L}$. The TPO Ab positivity had adverse effect on pregnancy even in situations where maternal TSH was within trimester specific normal range. Casey at al. ${ }^{13}$ in a large study of 17,298 pregnant woman found that the risk of premature delivery was not consistent as the increased risk of premature delivery at $<34$ weeks was $4 \%(\mathrm{p}=0.01)$ in compared to at $<32$ weeks $2.5 \%(\mathrm{p}=0.01)$ and $<36$ weeks $7 \%(\mathrm{p}=0.39)$. Similar adverse Pregnancy related outcomes were observed in meta analysis ${ }^{29}$ which have shown that TPOAb positivity in pregnant woman exacerbates pregnancy related complications.

The effect of maternal hypothyroidism on neurocognitive development of fetus is still unclear. Conflicting literature studies make things more difficult for clinicians in deciding treatment of maternal hypothyroidism solely to prevent fetal cognitive lag. Some studies showed reduction of IQ of children born to hypothyroid woman ${ }^{22}$ whereas others show no effect of subclinical hypothyroidism on motor skills and language development by comparing treatment and placebo. ${ }^{30-32}$ In these studies LT4 treatment was commenced after completion of I trimester whereas when treatment was started early as in animal studies ${ }^{33,34}$ significant impact on neuro cognitive milestones was noted. The conflicting literature reports are attributed to the fact that over past century iodine supplementation has been stressed by various countries and severe iodine deficiency is less commonly encountered rather Hashimoto's thyroiditis takes the driver's seat as etiology of maternal hypothyroidism. Study of Po et al. ${ }^{35}$ reported decreased psychomotor test scores with below normal FT4 levels in presence of normal serum TSH levels in woman. Li et al., ${ }^{36}$ and other studies ${ }^{12,37-39}$ observed low IQ, delay in language and poor motor function in children born to hypothyroid mothers. However there are limited studies in literature to show that LT4 administration improves or reduces harmful effects of maternal hypothyroidism on fetus. ${ }^{40-43}$ In a recent study by Korevaar et al. ${ }^{44}$ it was found that both low and high FT4 levels were associated with lower IQ of child and there was reduced cerebral gray matter volume in brain as assessed by MRI.

\section{Treatment}

The detrimental effects of maternal hypothyroidism have been shown in various studies leading to adverse outcome in pregnancy. ${ }^{45}$ There is lack of randomized control trial (RCT) to show occurrence of pregnancy loss in hypothyroid patients. A RCT demonstrated improved pregnancy related outcome in woman treated with LT4 since I trimester. ${ }^{46}$ In other studies, lower pregnancy loss was observed when TPO Ab positive woman who were biochemically euthyroid were treated with LT4. ${ }^{46,47}$ There is paucity of literature on trials involving TPOAb Negative woman. The current recommendation suggests that woman with elevated TSH should in addition undergo TPOAb status evaluation. A daily dose of $50 \mathrm{ug} /$ day is required for treatment of hypothyroidism.

LT4 therapy indications:

a. TPOAb positive with TSH greater than pregnancy specific reference range

b. TPOAb positive with TSH greater than $2.5 \mathrm{mU} / \mathrm{L}$ or age specific reference range and lower than upper limit of pregnancy specific range

\section{c. TPOAb Negative with TSH more than $10 \mathrm{Mu} / \mathrm{L}$}

LT4 therapy is not indicated for TPOAb negative woman with normal trimester specific TSH and for isolated Hypothyroxinemia. Oral LT4 therapy is recommended and T3 or desiccated thyroid should not be used. It is worth emphasizing that it is T4 that is required for developing fetal brain. ${ }^{48}$ The majority of fetal $\mathrm{T} 3$ is derived from maternal $\mathrm{T} 4$ as fetal $\mathrm{CNC}$ is impermeable to $\mathrm{T} 3$ containing preparation should be avoided during pregnancy. ${ }^{49,50}$ The target of treatment is to keep TSH below lower half of trimester specific reference range or in its absence below $2.5 \mathrm{mU} / \mathrm{L}$. Glinoer et al.$^{51}$ found that there is increased requirement of T4 during gestation and therefore TPOAb positive euthyroid woman might develop sub clinical hypothyroidism in later pregnancy.

Woman who have hypothyroid (treated or untreated), or post hemithyroidectomy or received radioactive iodine should undergo TSH measurements every 4 weeks till mid gestation and at least once at 30 weeks. Hypothyroid women planning or suspected or conformed for pregnancy should increase the dose of LT4 by 20$30 \%$ and inform physician for testing and evaluation. Patient should gradually taper off to preconceptional time dosing. A repeat thyroid function test is recommended at 6 weeks post partum while for those withnLT4<50ug/Day discontinuation is followed by TSH evaluation at 6 weeks is suggested.

\section{Conclusion}

Maternal hypothyroidsm is relatively common but frequently undiagnosed in pregnancy. The consequences on mother and fetus are detrimental. The guidelines by ATA present a lucid explanation for diagnosis and treatment of this ailment but it has to be seen and interpreted in context to local regional demographics of population.

\section{Acknowledgments}

None. 


\section{Conflicts of interest}

The author declares that there is no conflict of interest.

\section{References}

1. Stabnaro - Green A, Abalovich M, Alexander E, et al. American Thyroid Association Taskforce on Thyroid Disease During Pregnancy and Postpartum 2011Guidelines of the American Thyroid Association for the diagnosis and management of thyroid disease during pregnancy and postpartum. Thyroid. 2011;21:1081-1125.

2. Qaseem A, Snow V, Owens DK, Shekrlle P. The development of clinical practice guidelines and guidance statements of the American College of Physician. Summary of methods Ann Intern Med. 2010;153:194-199.

3. Van Raaij JM, Vermaat Miedema SH, Schonk CM, et al Energy requirements of pregnancy in the Netherlands. Lancet. 1987;2(8565):953-955.

4. Glinoer D. The regulationof thyroid function in malignancy: pathways of endocrine adaptation from physiology to pathology. Endocr Rev. 1997;18(3):404-433.

5. Weeke J, Dybkjaer L, Granlie K, et al. A longitudinal study of serum $\mathrm{TSH}$ and total and free iodothyronines during normal pregnancy. Acta Endocrinologica. 1982;101(4):531.

6. Baloch Z, Carayon P, Cone-Devolx B, et al. Guidelines Committee, National Academy of Clinical Biochemistry 2003 Laboratory medicine practice guidelines. Laboratory support for the diagnosis and monitoring of thyroid disease. Thyroid. 2003;13:3-126.

7. Soldin OP, Tractenberg RE, Hollowell JG, et al. Trimester-specific changes in maternal thyroid hormone, thyrotropin, and thyroglobulin concentration during gestation: trends and associations across trimester in iodine sufficiency. Thyroid. 2004;14(12):1084-1090.

8. Roti E, Gardini E, Minelli R, et al. Thyroid function evaluation by different commercially available free thyroid hormone measurement kits in term pregnant women and their newborns. $J$ Endocrionol Invest. 1991;14(1):1-9.

9. Sapin R, Herbomez M, Schlienger JL, et al. Free Thyroxine measured with equilibrium dialysis and nine immunoassays decreases in late pregnancy. Clin Lab. 2004;50:581-584.

10. Casey BM, Daashe JS, Wells CE, et al. Subclinical hypothyroidism and pregnancy outcomes. Obstet Gynecol. 2005;105(2):239-245.

11. Allan WC, Haddow JE, Palomaki GE, et al. Maternal thyroid deficiency and pregnancy complications :implications for population screening. J Med Screen. 2000;7(3):127-130.

12. Korevaar TL, Schalekamp-Timmermans S, de RijkeYB, et al. Hypothyroxinemia and TPO -antibody positivity are risk factors for premature delivery: the generation R study. J Clin Endocrinol Metab. 2013;98(11):4382-4390.

13. Casey BM, Dashe JS, Spong CY, et al. Perinatal significance of isolated maternal hypothyroxemia identified in the first half of pregnancy. Obstet Gynecol. 2007;109(5):1129-1135.

14. Haddow JE, Knight GJ, Palomaki GE, et al. The reference range and within -person variability of thyroid stimulating hormone during the first and second trimesters of pregnancy. J Med Screen. 2004;11(4):170-174.

15. Panesar NS, Li CY, Rogers MS. Reference intervals for thyroid hormones in pregnant Chinese women. Ann Clin Biochem. 2001;38:329-332.
16. Marwaha RK, Chopra S, Gopalkrishnan S, et al. Establishment of reference range for thyroid hormones in normal pregnant Indian women. BJOG. 2008;115(5):602-606.

17. Stricker R, Echenard M, Eberhart R, et al. Evaluation of maternal thyroid function during pregnancy: the importance of using gestational age specific reference intervals. Eur $J$ Endocrinol. 2007;157(4):509-514.

18. Soldin OP, Soldin D, Sastoque M. Gestation -specific thyroxine and thyroid stimulating hormone levels in the United States and worldwide. Ther Drug Monit. 2007;29(5):553-559.

19. Bocos - Terraz JP, Izquierdo -Alvarez Sm Bancalero - FloresJL, Alvarez - Lahuerta R, et al. Thyroid hormones according to gestation age in pregnant Spanish women. BMC Res Notes. 2009;2:237.

20. Medici M, KorevaarTI, Visser WE, et al. Thyroid function in pregnancy: what is normal? Clim Chem. 2015;61(5):704-713.

21. Van den Boogaard E, Vissenberg R, Land JA, et al. Significance of ( sub)clinical thyroid dysfunction and thyroid autoimmunity before conception and in the early pregnancy: a systematic review. Hum Reprod Update. 2011;17(5):605-619.

22. Hadow JE, Palomaki GE, Allan WC, et al. Maternal thyroid deficiency during pregnancy and subsequent neurophysiological development of the child. N Engl J Med. 1999;341(8):549-555.

23. Abalovich M, Gutierrez S, Alcaraz G, et al. Overt and subclinical hypothyroidism complicating pregnancy. Thyroid. 2002;12(1):63-68.

24. Leung AS, Millar LK, Koonings PP, et al. Perinatal outcome in hypothyroid pregnancies. Obstet Gynecol. 1993;81(3):349-353.

25. Wilcox AJ, Weinberg CR, O'Connor JF, et al. Incidnce of early pregnancy loss of pregnancy. N Engl j med. 1988;319(4):189-194.

26. Negro R, Schwartz A, Gismondi R, et al. Increased pregnancy loss rate in thyroid antibody negative women with TSH levels between 2.5 and 5.0 in the first trimester of pregnancy. $J$ Clin Endocrinol Metab. 2010;95(9):E44-E48.

27. Benhadi N, Wiersinga WM, Reitsma JB, et al. Higher maternal TSH levels in pregnancy are associated with increased risk for miscarriage, fetal or neonatal death. Eur J Endocrinol. 2009;160:985-991.

28. Schneuer FJ, Nassar N, Tasevski V, et al. Association and predictive accuracy of high TSH serum levels in first trimester and adverse pregnancy outcomes. $J$ Clin Endocrinol Metab. 2012;97(9):3115-3122.

29. Chan S, Boelaert K. Optimal management of hypothyroidism, hypothyroxinemia and euthyroid TPO antibody positivity preconception and in pregnancy. Clin Endocrinol (Oxf). 2015;82(3):313-326.

30. Casey BM. Effect of treatment of maternal subclinical hypothyroidism or hypothyroxinemia on IQ in offspring (Abstract). Ajog. 2017.

31. Liu H, Momotani N, Noh JY, et al. Maternal hypothyroidism during early pregnancy and intellectual development of the progeny. Arch Intern Med. 1994;154(7):785-787.

32. Downing S, Halpern L, Carswell J, et al. Severe maternal hypothyroidism corrected prior to the third trimester is associated with normal cognitive outcome in the offspring. Thyroid. 2012;22(6):625-630.

33. Auso E, Lavado-Autric R, Cuevas E, et al. A moderate and transient deficiency of maternal thyroid function at the beginning of fetal neurocorticogenesis alters neuronal migration. Endocrinol. 2004;145(9):4037-4047. 
34. Wang S, Teng W, Gao Y, et al. Early levothyroxine treatment on maternal subclinical hypothyroidism improves spatial learning of offspring in rats. J Neuroendocrinol. 2012;24(5):841-848.

35. Pop VJ, Bruwers EP, Vader HL, et al. Maternal hypothyroxinaemia during early pregnancy and subsequent child development: a 3 year follow-up study. Clin Endocrinol (Oxf). 2003;59(3):282-288.

36. Li Y, Shan Z, Teng W, et al. Abnormalities of maternal thyroid function during pregnancy affect neuropsychological development of their children at 25-30 months. Clin Endocrinol (OXF). 2010;72(6):825-829.

37. Karakotsa P, Alegakis D, Georgiou V, et al. Thyroid dysfunction and auto antibodies in early pregnancy are associated with increased risk of gestational diabetes and adverse birth outcomes. J Clin Endocrinol Metab. 2012;97(12):4464-4472.

38. Kumru P, Erdogdu E, Arisoy R, et al. Effect of thyroid dysfunction and autoimmunity on pregnancy outcomes in low risk population. Arch Gynecol Obstet. 2015;291(5):1047-1054.

39. Sahu MT, Das V, Mittal S, et al. Overt and subclinical thyroid dysfunction among Indian pregnant women and its effect on maternal and fetal outcome. Arch Gynecol Obstet. 2010;281(2):215-220.

40. Medici M, Timmermanns S, Visser W, et al. Maternal thyroid hormone parameters during early pregnancy and birth weight: the Generation R Study. J Clin Endocrinol Metab. 2013;98(1):59-66.

41. Leon G, Murcia M, Rebaagliato M, et al. Maternal thyroid dysfunction during gestation, preterm delivery and birthweight. The Infancia y Epidemiol. 2015;29(2):113-122.

42. Haddow JE, Craig WY, Neveux LM, et al. First and Secound Trimester Risk Of Aneuploidy (FaSTER) Research Consortium 2014 Implications of high free thyroxine (FT4) concentrations in euthyroid pregnancies : the FaSTER trial. J Clin Endocrinol Meta. 2014;99(6):2038-2044
43. Gyllenberg D, Sourander A, Surcel HM, et al. Hypothyroxinemia during gestation and offspring schizophrenia in a national birth cohort. Biol Psychiatry. 2016;79(12):962-970.

44. Korevaar TI, Muetzel R, Medici M, et al. Association of maternal thyroid function during early pregnancy with offspring IQ and brain morphology in childhood; A Population -based prospective cohort study. Lancet Diabetes Endocrinol. 2016;4(1):35-43.

45. Taylor PN, Minassian C, Rehman A, et al. TSH levels and risk of miscarriage in women on long-term levothyroxine: a community based study. J ClinEndocrinol Metab. 2014;99(10):3895-3902.

46. Negro R, Formoso G, Mangieri T, et al. Levothroxine treatment in euthyroid pregnant women with autoimmune thyroid disease: effects on obstetrical complications. J Clin Endocrinol Metab. 2006;91(7):2587-2591.

47. Lepoutre T, Debieve F, Gruson D, et al. Reduction of miscarriages through universal screening and treatment of thyroid autoimmune disease. Gynecol Obstet Invest. 2012;74(4):265-273.

48. Henrichs J, Ghassabian A, Peeters RP, et al. Maternal hypothyroxinemia and effects on cognitive functioning in childhood: how and why? Clin Endocrinol (Oxf). 2013;79(2):152-162.

49. Lev -Ran A. Part - of- the -day hypertriiodothyoninemia caused by dessicatedthyroid. JAMA. 1983;250(17):2790-2791.

50. Wiersinga W, Duntas L, Fadeyev V, et al. ETA GUIDELINES: the use of L-T4 +LT-3 in the treatment of hypothyroidism. Eur Thyroid J. 2012;1(2):55-71

51. Glinoer D, Velkeniers B, Anckaert E, et al. Thyroglobulin autoantibodies: Is there any added value in the detection of thyroid autoimmunity in women consulting for fertility treatment? Thyroid. 2013;23(8):1022-1028. 